\title{
Handlebar injuries in children
}

\author{
Peter Michael Klimek • Thomas Lutz • \\ Enno Stranzinger - Zacharias Zachariou • \\ Ulf Kessler • Steffen Berger
}

Accepted: 19 November 2012/Published online: 11 December 2012 (C) Springer-Verlag Berlin Heidelberg 2012

\begin{abstract}
Introduction Handlebar injuries in children may lead to severe organ lesions despite minimal initial signs and without visible skin bruise. We present our experiences applying a diagnostic and therapeutic algorithm for blunt abdominal trauma, and present the history of two selected cases.

Materials and methods We retrospectively assessed the charts of children below 16 years of age, only who were observed for $24 \mathrm{~h}$ or more in our institution due to a handlebar injury between 2004 and 2011. All children were treated according to an institutional algorithm.

Results 40 patients with a median age of 9.5 years were included. Diagnosed lesions were: ruptures of the liver $(n=6)$, spleen $(n=5)$, kidney $(n=1)$, and pancreas $(n=2)$, small bowel perforation $(n=3)$, and hernias of the abdominal $(n=2)$ or thoracic wall $(n=1)$. Surgical interventions were performed in 8 patients. The outcome was favorable in all the cases. Overall median hospitalization duration was 4.5 days (range 1-19 days). The overall duration between the accident and arrival at our emergency unit was $2.75 \mathrm{~h}$ (median, range 1-19 h). 20 children presented directly at our emergency unit after a median of $1.7 \mathrm{~h}$ (range 1-19.5 h). 20 children were referred by a family physician or a primary hospital after a median of $4.0 \mathrm{~h}$ (range 1-46 h).
\end{abstract}

P. M. Klimek (凹) · T. Lutz · Z. Zachariou · U. Kessler .

S. Berger

Department of Pediatric Surgery, Inselspital, University Hospital and University of Bern, 3010 Bern, Switzerland

e-mail: peter.klimek@insel.ch

E. Stranzinger

Department of Diagnostic, Interventional and Pediatric

Radiology, Inselspital, University of Bern, Bern, Switzerland
Conclusion Handlebar injuries in children resulted in serious trunk lesions in half of the present patient series. The spectrum of injuries in handlebar accidents varies widely, especially injuries to the abdomen can unmask often only in the course. We advocate close observation of patients with thoracic and abdominal handlebar injuries which may be regarded as blunt stab wounds. An institutional algorithm for blunt abdominal trauma management is supportive for emergency care in patients with handlebar injuries.

Keywords Handlebar injury · Children · Traumatic hernia

\section{Introduction}

Blunt abdominal trauma is one of the most frequent reasons for hospital admission following trauma in children [1]. Bicycle accidents are responsible for a significant part of trauma in children. Serious injuries mostly involve head and brain, associated with polytrauma from bicycle accidents or collisions with other vehicles. The second major injury mechanism is a direct handlebar impact resulting from lower speed crashes.

In case of a direct abdominal trauma, the abdominal organs of children are at a higher risk of organ lesions when compared to adults. This is due to a higher rate of transmission of forces through the abdominal wall and larger relative surfaces of the spleen and liver in children compared to adults [2]. Organ involvement in pediatric handlebar injuries is especially difficult to diagnose as external skin lesions may be missing. Additionally, pediatric patient complaints usually are more unspecific, resulting in a possible hazardous delay of diagnosis and treatment $[3,4]$. 
It has been shown that the application of institutional algorithms helps to establish a reliable diagnosis and to guide therapeutic decision [5-9, 11, 12]. Since 2004, we institutionalized an algorithm for diagnosis and treatment of hemodynamically stable blunt abdominal trauma (Fig. 1). Grading of organ lesions and treatment has been adapted according to Stylianos [5-9] and Strouse [10]. Primary treatment of those with an instable hemodynamic situation is not included in this algorithm but follows ATLS guidelines. In the present report, we aim to review the accuracy of diagnosis and subsequent treatment in the subgroup of patients with handlebar trauma.

\section{Materials and methods}

We reviewed the charts of 40 children under 16 years of age that were hospitalized for $24 \mathrm{~h}$ or more due to a direct handlebar impact to the trunk, between January 1st 2004 and December 31st 2011. All patients presented at the Interdisciplinary Pediatric Emergency Unit (Departments of Pediatrics and Pediatric Surgery, Inselspital, University Hospital Bern, Switzerland) either initially after the accident or secondarily after initial treatment in another institution.

All emergency physicians were trained according to the local guidelines including the algorithm (Fig. 1). However, patient management was not dictated by the algorithm, but at the discretion of the treating emergency team.

The study was approved by the local Children's Hospital ethical committee.

\section{Results}

From 2004 to 2011, among 134,116 children seen at our emergency department, 40 children (29 males, 11 females) were observed for $24 \mathrm{~h}$ or longer due to a handlebar injury. The median age was 9.5 years (range 3.5-16 years). Most accidents happened during summer time, with 27 out of 40 cases between June and September. In all patients, a direct impact of the handle bars into the body was

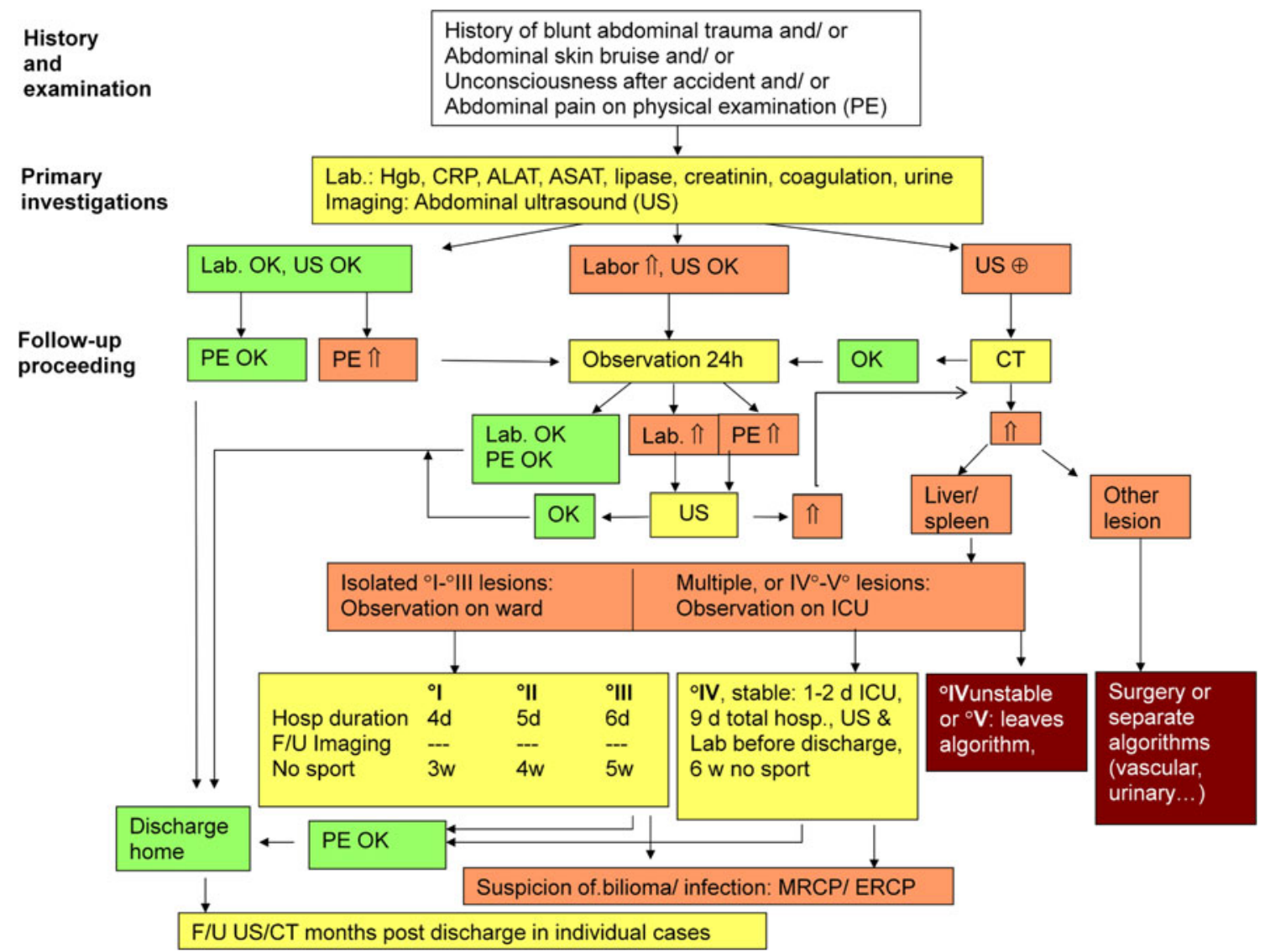

Fig. 1 Bernese algorithm for emergency management of stable children $<16$ years of age with isolated blunt abdominal trauma. $L a b$. laboratory investigations, $\mathrm{Hg} b$ hemoglobin, $O K$ result normal (not indicative of organ lesion or bleeding), up arrow result not normal (indicative of organ lesion or active bleeding), US ultrasound, $P E$ physical exam, $C T$ contrast enhanced computer tomography, ICU intensive care unit, $F / U$ follow-up, $d$ days, $w$ weeks 
documented. Symptom-free intervals were not described. All patients were hemodynamically stable without multiorgan injuries.

34 ultrasound examinations (in all patients with blunt abdominal trauma) and 6 radiographs were performed as initial imaging. Due to the pathologic ultrasound findings, 12 computed tomography studies and one MRI were performed. An overview of injuries is given in Table 1.

In 14 patients, a liver contusion was detected by pain in the upper abdomen and elevated liver enzymes: ASAT (Aspartate Aminotransferase), ALAT (Alanine Aminotransferase), without evidence of organ disruption in the ultrasound.

Potentially harmful lesions were found in 20 patients: ruptures of liver $(n=6)$, spleen $(n=5)$, pancreas $(n=2)$, kidney $(n=1)$, as well as small bowel perforation $(n=3)$ and abdominal and thoracic wall hernias $(n=3)$.

The overall duration between the accident and arrival at our emergency unit was $2.75 \mathrm{~h}$ (median, range 1-19 h). 20 children presented directly at our emergency unit after a median of $1.7 \mathrm{~h}$ (range 1-19.5 h). 20 infants were admitted by a family physician or a primary hospital after a median of $4 \mathrm{~h}$ (range 1-46 h).

32 of 40 patients were treated conservatively. 8 patients required surgical intervention. Surgically treated patients were primarily admitted to our institution (5 patients), the median duration between accident and intervention was $4 \mathrm{~h}$. In surgery patients that were secondarily transferred to our department ( 3 patients), the duration from accident to intervention was $1 \mathrm{~h}, 4 \mathrm{~h}$ and in one case $46 \mathrm{~h}$. The case with the longest delay presented with a complete rupture of the pancreas between the head and body which was diagnosed by CT at day 3 after the initial FAST ultrasound was normal. Partial resection of the pancreas was necessary in this case. The outcome was favorable in all the cases.
Overall median hospitalization duration was 4.5 days (range 1-19 days).

None of the patients that were discharged home without in-house observation returned because of abdominal problems. Also, there was no re-admission among those patients that were discharged after they had been hospitalized for diagnostics and observation according to the algorithm.

\section{Case reports}

The following two patient examples illustrate that a comparable anamnesis and the same initial symptoms may cause varying external skin- and internal organ lesions. The patients were hemodynamically stable and complained about abdominal pain, while examination revealed a tender abdomen. Hepatic and pancreatic enzyme levels as well as urine tests were normal (Figs. 2, 3).

\section{Discussion}

The present case series shows that pediatric handlebar injuries lead to serious lesions in a significant part of affected patients and a large variety of affected organs.

The spectrum of injuries we encountered (Table 1) is comparable to other studies in pediatric patients where parenchymatous organ lesions were found in up to $30 \%$, intestinal perforations in up to $10 \%[13,14]$, and traumatic abdominal wall hernia in up to $20 \%$ [15] and are also described in adults [16]. Traumatic thoracic wall hernias are very rare $[17,18]$.

The algorithm (Fig. 1) asks especially for a history of blunt abdominal trauma. It is important to differentiate
Table 1 Type of injury and treatment of patients with blunt abdominal trauma due to handlebar injury

\begin{tabular}{lccc}
\hline Type of injury & Number of patients & $\begin{array}{l}\text { Conservative } \\
\text { treatment }\end{array}$ & $\begin{array}{l}\text { Operative } \\
\text { treatment }\end{array}$ \\
\hline Contusion of the liver & 14 & 14 & \\
Hepatic rupture & 6 & 6 & \\
Splenic rupture & 5 & 5 & 1 \\
Rupture of the pancreas & 2 & 1 & \\
Rupture of the kidney & 1 & 1 & 3 \\
Perforation of the bowel & 3 & & 2 \\
Abdominal wall hernia & 2 & 1 & Recommended \\
Thoracic wall hernia & 1 & 2 & 1 \\
Bladder contusion & 2 & & \\
Sub-amputation of the finger & 1 & 1 & 1 \\
Hematoma in the abdominal wall & 1 & 1 & \\
Hematoma of the femoral vessel wall & 1 & 1 & \\
Rugged wound & 2 & & \\
\hline
\end{tabular}



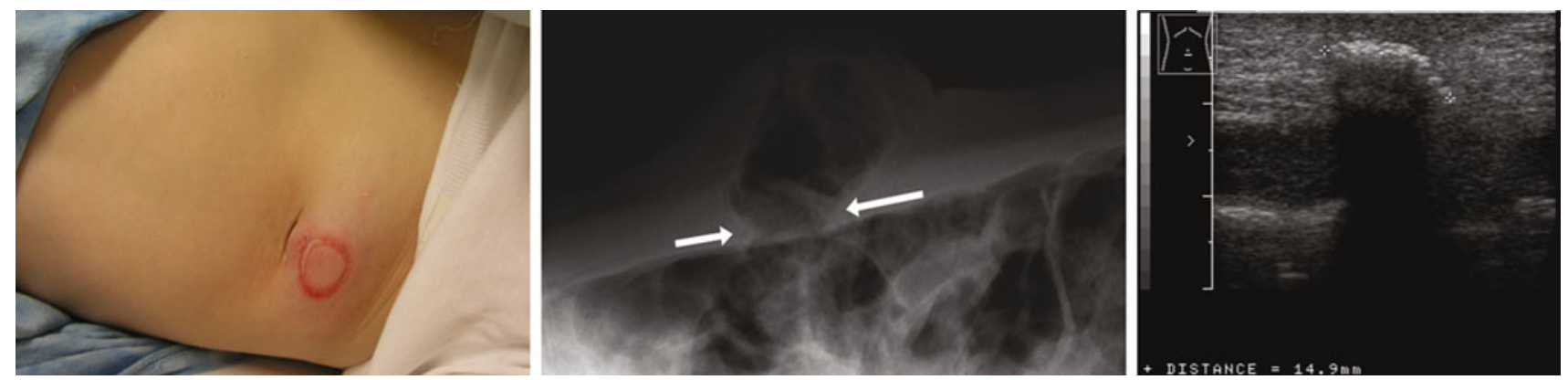

Fig. 2 12-year-old girl. Skin bruise after handlebar injury (left). Initial FAST ultrasound with gas bubbles in the subcutaneous region (right). A lateral radiograph of the abdomen confirmed a bowel herniation (arrow) through the abdominal wall (middle). Closure of the hernia was performed. The patient was discharged 4 days after surgery
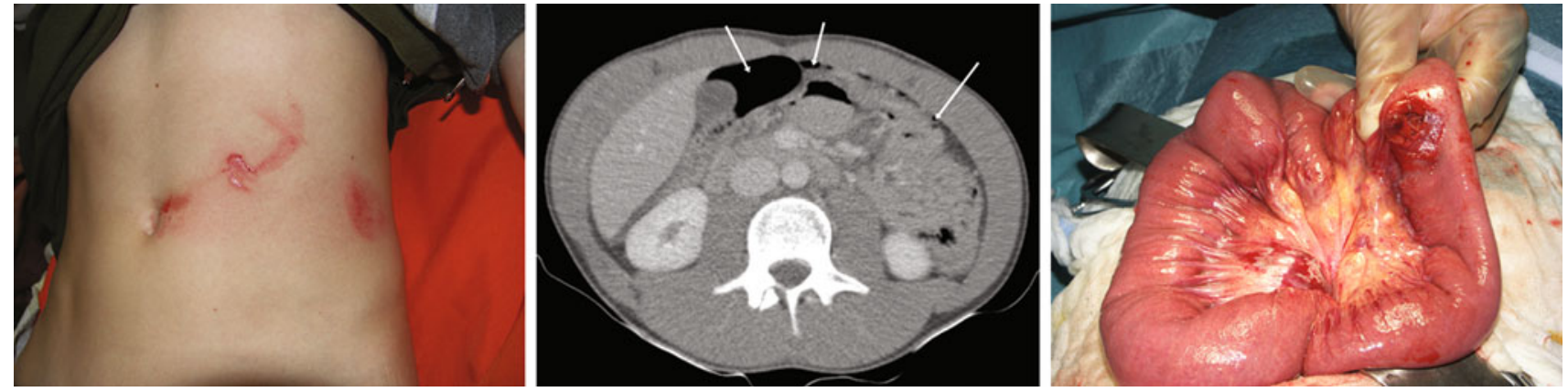

Fig. 3 15-year-old boy. Skin bruise after handlebar injury (left). Abdominal ultrasound revealed free retrovesical fluid and consecutive computed tomography (middle) showed free gas in the abdomen

between a simple fall from the bicycle (or flip over the handlebar) and direct impact to the abdomen. Organ injuries, length of hospital stay, and need for surgery has been shown to be increased in cases with direct impact from handlebars [3, 19].

In case of handlebar injuries, it is very important to hospitalize the patients in a hospital with the possibility for good pediatric monitoring, ultrasound, and computed tomography.

Additionally, it has been shown that delayed hospital presentation of patients with signs of more severe injury as e.g., strong abdominal pain following handlebar injury might lead to increased morbidity [20]. We therefore advocate the use of an algorithm also in peripheral hospitals or by family physicians to recognize patients with potentially severe intraabdominal injury for early referral to a tertiary hospital [21].

In the present study, the median duration from accident to hospital presentation or surgery was only slightly delayed in infants that were sent from other institution or a physician when compared to patients who initially presented at our institution but the range of 1-46 h was high.

The algorithm aims at directing the necessary amount of diagnostics to the individual patient with adequate safety and minimized radiation exposure. FAST ultrasound has (arrows) without further organ lesions. In the subsequent laparotomy a small bowel perforation (right) was closed. The patient was discharged 8 days after surgery

been introduced as a first step of imaging that can easily be repeated if symptoms persist and that can give (or withhold) the indication for an abdominal CT scan. Therefore, this algorithm might not only help in adjusting treatment following the result of a CT scan or the hemodynamic stability, as in previously suggested algorithms. The combination of a negative FAST and a negative blood test with a clinical improvement during $24 \mathrm{~h}$ surveillance gives a rather high safety to discharge the patient without a CT scan. On the other hand time delay for a necessary CT scan is minimal when a FAST detects an organ lesion or an age adjusted relevant amount of free fluid.

Hospital stay of patients with a confirmed spleen or liver injury, however, varies highly with regard to local habits. In many European pediatric centers, conservative management of abdominal solid organ injuries began early. Long periods of hospitalization were perceived as the prize for non-operative treatment and preservation of organs that otherwise might have been removed during the surgery. The short periods of hospitalization as recommended by Stylianos et al. in 2000 [6] are questioned in the meantime by possibly even shorter stays as suggested by St. Peter et al. in 2010 [22]. As the pendulum keeps swinging, the ideal compromise between patient safety and hospital resource utilization is not defined yet. 
In addition to the management of the following injury, the strategies for prevention may reduce subsequent morbidity in handlebar injuries. A great success for the prevention of severe head injuries by bicycle accidents was already achieved with the introduction of helmets [23], but security measures to prevent a direct handlebar trauma are almost completely absent. One possibility could be compressible handles or padded bar ends $[24,25]$. Other factors such as education of parents, children, and attending physicians might contribute to reduced morbidity.

We conclude that a high incidence of severe intraabdominal injuries is found in direct handlebar injuries in children. This trauma history should therefore be an indication for adequate imaging and in-house surveillance of patients at a pediatric trauma center which is prepared for surgical intervention and experienced with conservative treatment of organ injuries. Institutional algorithms may be helpful to improve the diagnostic process, decrease the rate of surgery, and shorten hospital stay.

Acknowledgments We thank Miriam Klimek and Mary Myrick for correction and translation.

Conflict of interest None of the authors has financial or personal relationships with other persons or organizations that could inappropriately influence his or her actions.

\section{References}

1. Winston FK, Weiss HB, Nance ML, Vivarelli-O'Neill C, Strotmeyer S, Lawrence BA et al (2002) Estimates of the incidence and costs associated with handlebar-related injuries in children. Arch Pediatr Adolesc Med 156(9):922-928

2. Schuetze U, Dietz R, Daum R, Kaboth H (1978) Die Fahrradlenkerverletung, eine Verletzung im Kindesalter. Unfallchirurgie 4(Nr.2):95-99

3. Nadler EP, Potoka DA, Shultz BL, Morrison KE, Ford HR, Gaines BA (2005) The high morbidity associated with handlebar injuries in children. J Trauma 58(6):1171-1174

4. Canty TG Sr, Canty TG Jr, Brown C (1999) Injuries of the gastrointestinal tract from blunt trauma in children: a 12-year experience at a designated pediatric trauma center. J Trauma 46(2):234-240

5. Stylianos S (1995) Controversies in abdominal trauma. Semin Pediatr Surg 4(2):116-119

6. Stylianos S (2000) Evidence-based guidelines for resource utilization in children with isolated spleen or liver injury. The APSA
Trauma Committee. J Pediatr Surg 35(2):164-167 (discussion 167-169)

7. Harris BH, Stylianos S (2001) Operative management of abdominal injuries in children. Semin Pediatr Surg 10(1):20-22

8. Stylianos S (2002) Compliance with evidence-based guidelines in children with isolated spleen or liver injury: a prospective study. J Pediatr Surg 37(3):453-456

9. Stylianos S (2005) Outcomes from pediatric solid organ injury: role of standardized care guidelines. Curr Opin Pediatr 17(3): 402-406

10. Strouse PJ, Close BJ, Marshall KW, Cywes R (1999) CT of bowel and mesenteric trauma in children. Radiographics 19(5):1237-1250

11. Nance ML, Keller MS, Stafford PW (2000) Predicting hollow visceral injury in the pediatric blunt trauma patient with solid visceral injury. J Pediatr Surg 35(9):1300-1303

12. Gross M, Lynch F, Canty T Sr, Peterson B, Spear R (1999) Management of pediatric liver injuries: a 13-year experience at a pediatric trauma center. J Pediatr Surg 34(5):811-816 (discussion 816-817)

13. Erez I, Lazar L, Gutermacher M, Katz S (2001) Abdominal injuries caused by bicycle handlebars. Eur J Surg 167(5):331-333

14. Sparnon AL, Ford WD (1986) Bicycle handlebar injuries in children. J Pediatr Surg 21(2):118-119

15. Karaman I, Karaman A, Aslan MK, Erdoğan D, Cavuşoğlu YH, Tütün O (2009) A hidden danger of childhood trauma: bicycle handlebar injuries. Surg Today 39(7):572-574

16. Prada Arias M, Dargallo Carbonell T, Estévez Martínez E, Bautista Casasnovas A, Varela Cives R (2004) Handlebar hernia in children: two cases and review of the literature. Eur J Pediatr Surg 14(2):133-136

17. Wittenberger R, Elias K (1975) Thoracic-wall hernia with pulmonary prolapse due to injury. Zentralbl Chir 100(16):1003-1005

18. Maeda R, Isowa N (2008) Traumatic intercostal pulmonary hernia; report of a case. Kyobu Geka 61(6):504-507

19. Shah S, Sinclair SA, Smith GA, Xiang H (2007) Pediatric hospitalizations for bicycle-related injuries. Inj Prev 13(5):316-321

20. Lam JP, Eunson GJ, Munro FD, Orr JD (2001) Delayed presentation of handlebar injuries in children. BMJ 322(7297): $1288-1289$

21. Hall JR, Reyes HM, Meller JL, Loeff DS, Dembek R (1996) The outcome for children with blunt trauma is best at a pediatric trauma center. J Pediatr Surg 31(1):72-76 (discussion 76-77)

22. St Peter SD, Sharp SW, Snyder CL, Sharp RJ, Andrews WS, Murphy JP, Islam S, Holcomb GW 3rd, Ostlie DJ (2011) Prospective validation of an abbreviated bedrest protocol in the management of blunt spleen and liver injury in children. J Pediatr Surg 46(1):173-177

23. Parkin PC, Howard AW (2008) Advances in the prevention of children's injuries: an examination of four common outdoor activities. Curr Opin Pediatr 20(6):719-723

24. Kubiak R, Slongo T (2003) Unpowered scooter injuries in children. Acta Paediatr 92(1):50-54

25. Clarnette TD, Beasley SW (1997) Handlebar injuries in children: patterns and prevention. Aust N Z J Surg 67(6):338-339 